\title{
Neue Forschungsansätze über Theaterkultur in österreichischen Prälatenklöstern der Frühen Neuzeit
}

\section{Recent Scholarship and New Methods of Research concerning Monastic Performance and Theatricality in the Baroque Era}

Alkuin Volker Schachenmayr / alkuin.schachenmayr@hochschule-heiligenkreuz.at Benedict XVI Philosophical-Theological University, Heiligenkreutz, AT

\begin{abstract}
Research about musical and theatrical performance in baroque men's and women's monasteries has developed significantly since 2000. After noting some major publications, this article points to the most important archival sources in the liturgical and economic areas that are crucial for understanding monastic performance culture. A conference held recently in Vienna about the history of liturgy and performance during Holy Week served as an impetus for further work in this area.
\end{abstract}

\section{Keywords}

liturgy, Holy Week, monks and nuns in the performing arts, prelatial monasteries of the Habsburg Empire, history of daily life 
Die Geschichte des kirchlichen Theaters in der Barockzeit darf nicht auf das Jesuitentheater reduziert werden. ${ }^{1}$ Diese Engführung würde übersehen, dass viele andere Orden außer der Gesellschaft Jesu als Theatermacher aktiv waren. Ebenso verengt das Bild des "Jesuitentheaters" den Theaterbetrieb auf die Schulbühne; somit fällt die Vielfalt der Performanz-Gattungen, die in der kirchlichen Öffentlichkeit regelmäßig vorkamen, aus dem Sichtfeld der Forschung. Neue Zugänge zur Erforschung der klösterlichen Theaterkultur bieten sich in jüngeren Publikationen an. Veränderungen in der theaterwissenschaftlichen Analyse dieser Quellen sollen hier skizziert werden.

Im Verlauf des 20. Jahrhunderts erschienen immer mehr Forschungsergebnisse über diverse Arten von Klostertheater. Üblicherweise genossen nach den Jesuiten die Benediktinerschulen die meiste theaterwissenschaftliche Aufmerksamkeit. ${ }^{2}$ Die von Heinz Kindermann initiierte und von der Österreichischen Akademie der Wissenschaften herausgegebene Buchreihe zur Theatergeschichte Österreichs publizierte beispielsweise Monographien über das Theater an der Benediktineruniversität in Salzburg und die Theaterkultur des Stifts Seitenstetten. Diese Studien aus früheren Generationen sind auf notwendige Grundlagen wie Spielplan- und Personenforschung eingegangen. Mit der Erscheinung des Buches von Freeman über die barocke Musikkultur im Stift Melk ${ }^{3}$ war eine wichtige Ergänzung in Richtung Musikwissenschaft gemacht: Freeman betonte auf unmissverständliche Weise die grundlegende Verbindung zwischen Musik und Theater; es gibt kein Theater ohne Musik.

Ein nächster Erkenntnisschritt kam mit der Erfassung der darstellenden Kunst bei Nonnen. Das Ausmaß der musiktheatralischen Aufführungen in Frauenklöstern war vielen unbekannt, als kurz nach 2000 zwei wichtige Bücher darüber erschienen. ${ }^{4}$ Leider nahmen sie keinen Bezug zum mitteleuropäischen Milieu. Die 2014 gedruckte Monographie von Janet Page füllte diese Lücke mit einer engen Fokussierung und reichen Auswertung der Lage in Wiener Frauenklöstern des 18. Jahrhunderts. ${ }^{5}$

An dieser Stelle soll Frank Pohles 2010 veröffentlichte Dissertation über jesuitisches Schultheater in einigen deutschen Ländern als methodologisches Modell genannt werden, auch wenn Prälatenklöster dort nur am Rande vorkommen. Vorbildlich an Pohles Buch ist seine breitgefächerte Reflexion über die Einbindung des theatralischen Momentes mit der geistlichen Trägerinstitution in all ihrer Komplexität. Er schöpft aus einer Quellenlage, die weit über die Periochen hinaus geht und behandelt einen beacht-

1 MÜLLER, Johannes. Das Jesuitendrama in den Ländern deutscher Zunge vom Anfang (1555) bis zum Hochbarock (1665). Augsburg: Filser, 1930.

2 HALLER, Edmund. Grundzüge einer Bibliographie des Schuldramas zu Kremsmünster. Heimatgaue. 1927, vol. 8, p. 324-329. Vom Verfasser sind viele Publikationen über die Theatergeschichte österreichischer Klöster erschienen.

3 FREEMAN, Robert. The Practice of Music at Melk Abbey. Based upon the Documents 1681-1826. Wien: Verlag der Österreichischen Akademie der Wissenschaften, 1989.

4 REARDON, Colleen. Holy Concord within Sacred Walls. Nuns and Music in Siena, 1575-1700. Oxford: Oxford University Press, 2002. WEAVER, Elissa. Convent Theatre in Early Modern Italy. Spiritual Fun and Learning for Women. Cambridge: Cambridge University Press, 2002.

5 PAGE, Janet. Convent Music and Politics in Eighteenth-Century Vienna. Cambridge: Cambridge Universtiy Press, 2014. 
lichen Reichtum: Einzugsgebiete, konfessionelle Fragen, Reformbestrebungen innerhalb eines Ordens oder Fragen der Ablöse eines Ordens durch einen anderen, systematische Untersuchungen der Rhetorik und des dramaturgischen Regelwerkes, Stücke- und Stoffwanderung, Gattungszwischenräume wie „szenische Deklamationen“, Kanonbildung, die vielen Spielanlässe vom Heiligenfest zum Fürstbesuch, Spielorte, Bühnenbauten, Spielzeiten, Spielvorbereitung, Personengruppen (Träger, Schauspieler, Musiker, Publikum und Stifter) und die Nebenformen der Performanz wie Prozessionen, Katechesen, Volksmissionen, Trauerkulissen und Heilige Gräber. In seinem Eifer geht der Verfasser dieses mehr als tausendseitigen Buches auch auf Mendikanten und - an vorletzter Stelle - Benediktiner ein. ${ }^{6}$

Diese und ähnliche Forschung seit dem Jahr 2000 lässt erkennen, dass die Theater- und Musikaufführungen in den Prälatenklöstern - jenen traditionsreichen „Stiften“ mit Grundherrschaft, niederer Gerichtsbarkeit und Stimme in den Landständen - seit Jahrzehnten nicht erforscht wurden. Das Interesse von österreichischen Theaterwissenschaftlern hat sich von den Klöstern abgewendet. Über die häufig lateinisch gespielten Stücke mit heidnisch-antiker oder christlicher Thematik werden an den Fakultäten selten Dissertationen geschrieben. Anregungen für dieses Gebiet kommen aus Studien zur Symbolik, Kommunikation und Zeremoniell. Besonders verheißungsvoll ist der „Sonderforschungsbereich 496 (Münster)“ zur symbolischen Kommunikation und zu gesellschaftlichen Wertesystemen. Diese Forschung aus der Ferne (ihre Betreiber identifizieren sich selten als Theaterwissenschaftler im engeren Sinn) regt dazu an, die Prälatenklöster neu zu entdecken. Die liturgische und ästhetische Vielfalt der klösterlichen Theaterereignisse erstreckt sich über das ganze liturgische Jahr und ist eine verhältnismäßig geschlossene Subkultur, deren Archivalien mancherorts gut erhalten sind. Jedenfalls sind Männerklöster fast immer besser dokumentiert als diejenigen von Frauen, also wären noch reichere Studien möglich, als die kürzlich von Reardon, Weaver und Page vorgelegten. Die Prälatenklöster stellen ein großes, aber geschlossenes Gefüge dar, in dem weitläufige Grundherrschaft, kirchliche Selbstpräsentation und Frömmigkeit sich gegenseitig beeinflussen.

Ein 2018 lancierter Forschungsansatz im Umfeld von Wien nimmt sich der Karwoche als Verdichtungsmoment an. Am 15./16. März dieses Jahrs fand ein interdisziplinärer Workshop über die Kreuzestheologie und Passionsfrömmigkeit in der Residenzstadt Wien von ca. 1500 bis 1835 statt. Veranstalter waren das Don Juan Archiv und das Institut für kunst- und musikhistorische Forschungen der Österreichischen Akademie der Wissenschaften. Die Auswahl der Karwoche als Zeitpunkt rechtfertigt sich durch die Stellung dieser acht Tage als Höhepunkt des katholischen Jahreskreises. In ihr verdichten sich diverse Gattungen darstellender Kunst. Ein theatralischer Beitrag der Karwoche in den Prälatenklöstern war das Karfreitagsspiel, das in einer Kirche vor dem Heiligen Grab gespielt wurde. Die weit verbreitete Spielgattung hielt sich als quasi-kanonischer Bestandteil der Woche über hundert Jahre. Wir dürfen vermuten, dass ein neuer Text jedes Jahr erstellt wurde, obwohl Stoffwiederholungen denkbar sind. Die heute erhaltenen

6 POHLE, Frank. Glaube und Beredsamkeit. Katholisches Schultheater in Jülich-Berg, Ravenstein und Aachen (1601-1817). Münster: Rhema-Verlag, 2010. 
Karfreitagsspiele aus dem Stift Heiligenkreuz zum Beispiel beginnen im Jahr 1661 und reichen bis 1759. Die Überlieferung für die Zeit von 1731 bis 1759 ist besonders dicht. Während dieser Jahre waren die Prälatenklöster in und um Wien gut vernetzt; die personale Verbindung des Stiftes Heiligenkreuz zur Wiener Hofmusikkapelle durch die Reutter Familie ist ein Beispiel unter vielen. ${ }^{7}$ Daher gehören die Karfreitagsspiele in den Klöstern Klosterneuburg, Schottenstift, Melk und Heiligenkreuz zu einer kulturhistorischen Auseinandersetzung mit der Karwoche in Wien, auch wenn die Klöster nicht alle innerhalb der Stadtgrenze liegen. Trotzdem hielten sie prominente Stadthöfe in Wien, sie engagierten Musiker aus der Residenzstadt und der Kaiserhof hielt sich regelmäßig in den Prälatenklöstern auf, unter anderem um dort Theaterabende zu genießen.

Was sollte ein neuer Forschungsansatz berücksichtigen, um das Theater der Karwoche und ähnliche Phänomene zu analysieren? Um mit dem wichtigsten anzufangen: Die religiöse Komponente, die sich in Bibel und liturgischen Texten aus den Messproprien, Präfationen und Tagesgebeten zusammensetzt, darf nicht ausgeklammert werden. Diese Texte waren den Religiosen zeitlebens vertraut und kehrten im kalendarischen Rhythmus wieder. Rhetorische Anklänge sind in den Theaterstücken mit Sicherheit zu erwarten. Gewisse Dinge waren für die Mönche und ihr Publikum selbstverständlich, sind es aber heute nicht mehr: Dass die öffentliche Fußwaschung am Gründonnerstag die Nachahmung einer Handlung Jesu Christi ist oder dass die Zwölferzahl der Gruppe, deren Füße gewaschen werden, auf das Apostelkollegium bezogen ist. Ebenso sollte man einen Blick auf spirituelle Handbücher, die von Exerzitienmeistern und Beichtvätern als Leitfaden der Betrachtung verwendet wurden, richten: Paraliturgica wie Speiseweihen oder Kreuzwegbetrachtungen in Dialogform sind ebenso aufschlussreich.

Normative liturgische Quellen sind zwar mit Vorsicht zu behandeln, weil die alltägliche Praxis von ihnen erheblich abweichen kann, dennoch geben sie stets eine unentbehrliche Orientierung. Das richtige offizielle Ritualbuch zu finden, ist nicht immer einfach, da es nicht nur römische Ausgaben gibt, sondern jeweils eigene für jeden Orden (Prämonstratenser, Benediktiner, Cistercienser usw.). Freilich ist damit nicht gesagt, dass sie im jeweiligen Kloster auch verwendet wurden, da der Trend in der Barockzeit zur römischen Vereinheitlichung ging. Schließlich gibt es diesbezügliche Archivquellen, nämlich handgeschriebene Hausritualien. Dort wo diese erhalten sind, sollten sie besonders genau ausgewertet werden.

Die Publikumsforschung des Klostertheaters ist so gut wie gar nicht erschlossen, sieht man vom Personenkreis des Hofes ab. Innerhalb des Prälatenklosters eröffnete sich eine eigene feudale Welt mit verschiedenen Ständen. Im Konvent gab es die Stände der Priestermönche, Chormönche, Konversen, Familiare und Sängerknaben. Angestellte im höheren Bereich (Arzt, Apotheker, Organist, Regens chori) gehörten zwar nicht dem Konvent an, waren aber generell klausurberechtigt. Außerhalb der Klausur gab es weitere Kreise, die nicht geschlossen waren, aber trotzdem einheitlich zu verstehen sind: Die Arbeiter in Küche und Wäscherei, sowie die landwirtschaftlichen Handwerker in der Umgebung des Stiftes. Wiederholungen kommen in den inkorporierten Pfarreien des

7 GLÜXAM, Dagmar und FASTL, Christian. Art. Reutter, Familie. Österreichisches Musiklexikon [online], [cit. 2018-03-28]. URL: https://www.musiklexikon.ac.at/ml/musik_R/Reutter_Familie.xml. 
Stiftes vor, die strukturell mehr als Grundherrschaft und weniger als seelsorgliche Räume verstanden werden müssen. Bewegung war unter diesen Dörfern durchaus möglich, vor allem zu theaterfreundlichen Anlässen. Die Gruppen waren alle anders genormt und sind daher in der Analyse anders zu betrachten.

Chroniken des Klosterlebens sind in Tagebüchern der Mönche erhalten, werden aber selten erforscht, weil sie meist inhaltlich nicht erschlossen oder verzeichnet sind. Ebenso sind Prioratschroniken und Abtskalender vorhanden, um Details über Aufführungspraxis zu gewinnen. In manchen besonders kostbaren Fällen sind sogar mehrere Mönche gleichzeitig als Tagebuchschreiber tätig - daraus entsteht ein Vergleichspotential.

Wirtschaftsakten wie Küchenrechnungen oder Kammeramtsrechnungen erschlieBen die Vorbereitungszeit eines festlichen Anlasses und bezeugen die Geldsummen, die dafür ausgegeben wurden. Rechnungen sind realistisch, wobei normative Quellen im theoretischen Bereich und Egodokumente im subjektiven Empfinden zurückbleiben. Geldaufwand sagt wesentlich konkreter aus, welches Gewicht ein Fest in der Wirklichkeit des Alltags hatte. Theatralische Inszenierungskosten und Küchenrechnungen sind meist komplementär, auch wenn Küche und Bühne nicht direkt miteinander in Kontakt treten.

Der Forscher muss auch über das Stiftsarchiv hinausschauen, und zwar auf andere zeitgenössische, kirchliche Quellen. Bischöfliche Weihebücher und Protokollhefte eines Zeremoniärs erlauben Rückschlüsse auf die Klöster, weil die Priester eines Klosters die niederen und hohen Weihen meist in der Bischofskirche oder -kapelle empfingen. Ordensmänner treten recht häufig im diözesanen Archivgut der Frühen Neuzeit auf. Eine Quelle wie das bischöfliche Weihebuch reflektiert wiederum von einer anderen Perspektive auf das Kirchenjahr. Heute würden wir nie erwarten, dass eine Priesterweihe am Karsamstag stattfindet! Die Grabesruhe Jesu Christi und die Sehnsucht nach der bevorstehenden Ostervigil prägen das heutige Empfinden, aber in der Tat haben Bischöfe im 18. Jahrhundert die Priesterweihe oft an diesem Tag gespendet. Nur Stunden zuvor zogen Bruderschaften durch die Straßen der Bischofsstadt und geißelten sich. So grundlegend kann man eine Stimmungslage verschätzen.

Ein Proprium der Theaterkultur in und um die Prälatenklöster wurde bereits von frühen Forschern erkannt: Das Publikum scheint heterogener gewesen zu sein als im Schultheater der Jesuiten. Weil im Stiftstheater das Publikum aus Höflingen oder auch Knechten bestehen konnte, macht sich eine Tonvielfalt vom religiös Erhabenen zum ordinär Erheiternden bemerkbar. Jesuitentheater wurde fast immer in lateinischer Sprache gespielt. Das Mönchstheater zeigte sich viel offener für die Volkssprache, ja sogar die „derbe Mundart“ und eine lokale Inspiration „aus dem Volke“ ist Forschern vor Generationen bereits aufgefallen. ${ }^{8}$ Jüngere Forschung aus Oberösterreich greift diese Pfade enthusiastisch auf. ${ }^{9}$

Schlussendlich sei vor positivistischer Überkategorisierung gewarnt: Die formelle Unterscheidung zwischen Sepolcro und Oratorium, Dreikönigsspiel und Faschingsallegorie

8 HALLER, op. cit., p. 324.

9 NEUHUBER, Christian. Maurus Lindemayr, Dialektlieder. Kritische Ausgabe Bd. 2, Kommentar. Wien: Praesens-Verlag, 2008. 
lässt sich nicht immer verallgemeinern und kann viel Energie verschwenden. Sogar die Gattung des Karfreitagsspiels ist nicht immer gesichert. Es wurde zwar am Karfreitag gespielt und heißt auch deswegen zu recht so, aber der Begriff bietet darüber hinaus kaum eine brauchbare inhaltliche Präzisierung, denn diese Stücke können sich in der narrativen Struktur, Themen und im Ton radikal voneinander unterscheiden.

Verheißungsvollere Zugänge könnten sich anstatt obiger Klassifikationen eher mit den Ordnungskriterien Darsteller, (Bühnen-)Bild und Raum befassen. Grundlegende Arbeit über den liturgischen und zeremoniellen Aspekt der Inszenierung ist schon von Ursula Brosette geleistet worden. ${ }^{10}$ Da der Darsteller selten ein Berufsschauspieler war, muss er im Geflecht von Schule oder seinem ökonomischen Verhältnis zum Stift verstanden werden. Das Bild, vor dem gespielt wurde, bestand nicht selten aus Sakralarchitektur. Bei der Frage nach dem Raum sind die Möglichkeiten oft verkürzt präsentiert worden. In Kirchenräumen kann gespielt worden sein, aber auch im Refektorium, in der Prälatur, im Innenhof oder auf Emporen.

Da die geschichtswissenschaftliche Auseinandersetzung mit dem Theater in den letzten Jahren generell nach neuen Paradigmen sucht, wäre es gewinnbringend, das Klostertheater der Stifte wieder und gründlicher aufzugreifen. Das religiös-kultische Moment ist zwar in der öffentlichen Theaterarbeit unserer Zeit aktuell, aber nicht in Bezug auf katholische Klöster und ihre Künstler. Internationale und interdisziplinäre Forschungsansätze haben gezeigt, dass sich eine neue Methodologie bewährt und wertvolle Erkenntnis bringt.

\section{Bibliography}

BROSSETTE, Ursula. Die Inszenierung des Sakralen. Das theatralische Raum- und Ausstattungsprogramm süddeutscher Barockkirchen in seinem liturgischen und zeremoniellen Kontext. 2 Bände. Weimar: VDG, 2002.

FREEMAN, Robert. The Practice of Music at Melk Abbey. Based upon the Documents 1681-1826. Wien: Verlag der Österreichischen Akademie der Wissenschaften, 1989.

GLÜXAM, Dagmar und FASTL, Christian. Art. Reutter, Familie. Österreichisches Musiklexikon [online], [cit. 2018-03-28]. URL: https://www.musiklexikon.ac.at/ml/musik_R/Reutter_Familie. $\mathrm{xml}$.

HALLER, Edmund. Grundzüge einer Bibliographie des Schuldramas zu Kremsmünster. Heimatgaue. 1927, vol. 8, p. 324-329.

MÜLLER, Johannes. Das Jesuitendrama in den Ländern deutscher Zunge vom Anfang (1555) bis zum Hochbarock (1665). Augsburg: Filser, 1930.

NEUHUBER, Christian. Maurus Lindemayr, Dialektlieder. Kritische Ausgabe Bd. 2, Kommentar. Wien: Praesens-Verlag, 2008.

PAGE, Janet. Convent Music and Politics in Eighteenth-Century Vienna. Cambridge: Cambridge Universtiy Press, 2014.

10 BROSSETTE, Ursula. Die Inszenierung des Sakralen. Das theatralische Raum- und Ausstattungsprogramm süddeutscher Barockkirchen in seinem liturgischen und zeremoniellen Kontext. 2 Bände. Weimar: VDG, 2002. 
POHLE, Frank. Glaube und Beredsamkeit. Katholisches Schultheater in Jülich-Berg, Ravenstein und Aachen (1601-1817). Münster: Rhema-Verlag, 2010.

REARDON, Colleen. Holy Concord within Sacred Walls. Nuns and Music in Siena, 1575-1700. Oxford: Oxford University Press, 2002.

WEAVER, Elissa. Convent Theatre in Early Modern Italy. Spiritual Fun and Learning for Women. Cambridge: Cambridge University Press, 2002. 
